\title{
SURFACE MICROMACHINED PIEZOELECTRIC RESONANT BEAM FILTERS
}

\author{
Brett Piekarski, ${ }^{1,2}$ Don DeVoe, ${ }^{2}$ Madan Dubey, ${ }^{1}$ Roger Kaul, ${ }^{1}$ John Conrad, ${ }^{1}$ Robert Zeto ${ }^{1, *}$ \\ ${ }^{1}$ U.S. Army Research Laboratory Adelphi, Maryland 20783-1197 \\ ${ }^{2}$ Dept. of Mechanical Engineering and Institute for Systems Research \\ University of Maryland College Park, Maryland 20742
}

\begin{abstract}
Piezoelectric actuation and sensing of suspended single-beam microelectromechanical system (MEMS) resonant filters is demonstrated. Resonant frequencies between $171 \mathrm{kHz}$ and 9.2 MHZ were observed for resonators with dimensions of 6- to 20 $\mu \mathrm{m}$ wide and $25-$ to $400-\mu \mathrm{m}$ long. Operation was demonstrated from a vacuum pressure of 20 -mTorr to atmospheric with drive voltages as low as $2.5 \mathrm{mV}$ and a dynamic range of greater than 40 $\mathrm{dB}$. Loaded quality factors (Qs) greater than 1000 resulted at 9.2 $\mathrm{MHz}$ and a 20 -mTorr pressure. The resonators utilize a clampedclamped beam design with sol-gel lead zirconate titanate (PZT) as the piezoelectric actuator and sensor, PECVD silicon dioxide as the resonant beam structure, and platinum as the top and bottom electrode.
\end{abstract}

\section{INTRODUCTION}

Due to their small size, low power consumption, and ability to be integrated with microelectronics, MEMS resonators are becoming viable for use as filters in communication systems. To date, these efforts have focused primarily on using electrostatic transduction for actuation and sensing of polysilicon and silicon resonant beams $[1-5]$.

The theoretical coupling strength of electrostatic devices has been shown to decay rapidly as resonant frequencies are increased. Piezoelectric transduction offers an alternative mechanism for micromachined resonant clamped-clamped beams that avoids this difficulty, since piezoelectric coupling strength is significantly higher than electrostatic coupling and decays more slowly as beam dimensions are decreased to increase the resonant frequency [6]. Reduction in coupling strength is slower because capacitive actuation results from a distributed force whereas piezoelectric actuation results from a distributed moment independent of the beam length. Improved coupling can potentially lead to improved performance and reduced noise.

Piezoelectric resonators demonstrated with $\mathrm{ZnO}$ as the piezoelectric actuation and sense material were performance limited by the low piezoelectric coefficients of $\mathrm{ZnO}$ [6]. PZT has much higher piezoelectric coefficients and while devices using PZT as the piezoelectric material have been used as actuators and force sensors [7-12], little work has been reported on thin-film PZT resonators for communication systems. Here, we demonstrate suspended single-beam piezoelectric clamped-clamped micromechanical resonators using sol-gel-deposited PZT as the active piezoelectric for both sensing and actuating beam resonance.

\section{RESONATOR STRUCTURE AND FABRICATION}

Figure 1 shows a top view and cross section of the clampedclamped beam resonator design. The starting wafer is fabricated by first depositing a PECVD $\mathrm{SiO}_{2}$ layer, underlies the entire structure, on a Si wafer.
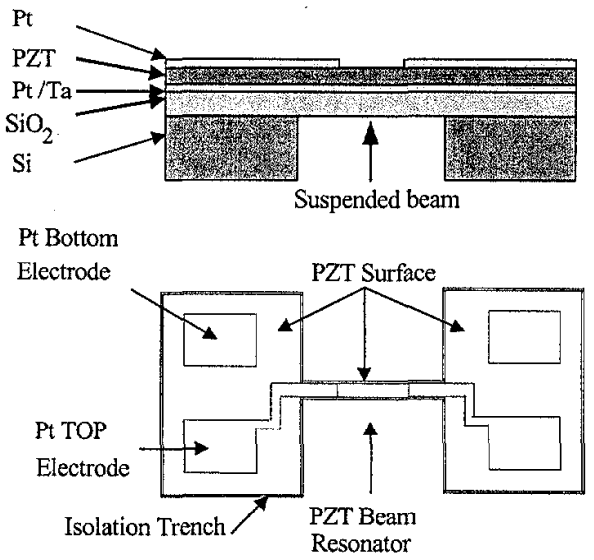

Figure 1. Resonator design.

A sputtered tantalum adhesion layer is then deposited on top of the oxide, followed by a sputtered platinum bottom electrode that also underlies the entire structure. The piezoelectric material is a sol-gel $\mathrm{PZ}_{52} \mathrm{~T}_{48}$, spun on and pyrolyzed until the desired PZT thickness is achieved. A sputtered platinum top electrode layer is then deposited over the PZT. Table 1 shows the thickness of each layer used in the reported devices.

Table 1. Material thickness.

\begin{tabular}{|c|c|}
\hline Material & Thickness $(\mu \mathrm{m})$ \\
\hline Silicon Dioxide & 2 \\
\hline Tantalum & 0.02 \\
\hline Bottom Platinum & 0.17 \\
\hline PZT & 0.5 \\
\hline Top Platinum & 0.17 \\
\hline
\end{tabular}

Resonator fabrication starts by defining the top electrode pattern through photolithography and ion milling away the unwanted platinum. An opening is then created in the PZT by wet etching to provide access to the bottom electrode. The beam length and width are then defined, along with an electrical isolation

* Currently with General Technical Services, Wall Township, NJ 07719 
trench, by ion milling through the $\mathrm{PZT}$, bottom electrode, and $\mathrm{SiO}_{2}$ to expose the underlying silicon. The final step is an isotropic silicon reactive-ion etch (RIE) that undercuts the beam geometry and results in the suspended clamped-clamped beam. Additional photolithography can be done prior to the isotropic silicon RIE step so that the isotropic etch undercuts only the photoresist and not the beam anchors while the beam is undercut. Figures 2 and 3 are SEM micrographs of a 50- $\mu \mathrm{m}$ long beam and show the isolation trench, probe contact pads, isotropically etched trench, and the quarter length top electrodes.

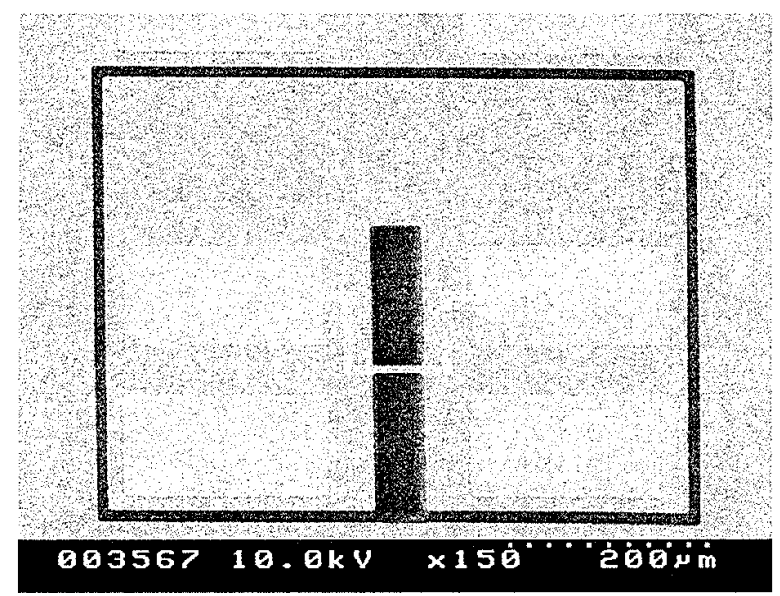

Figure 2. Top view of 50- $\mu \mathrm{m}$ long resonator.

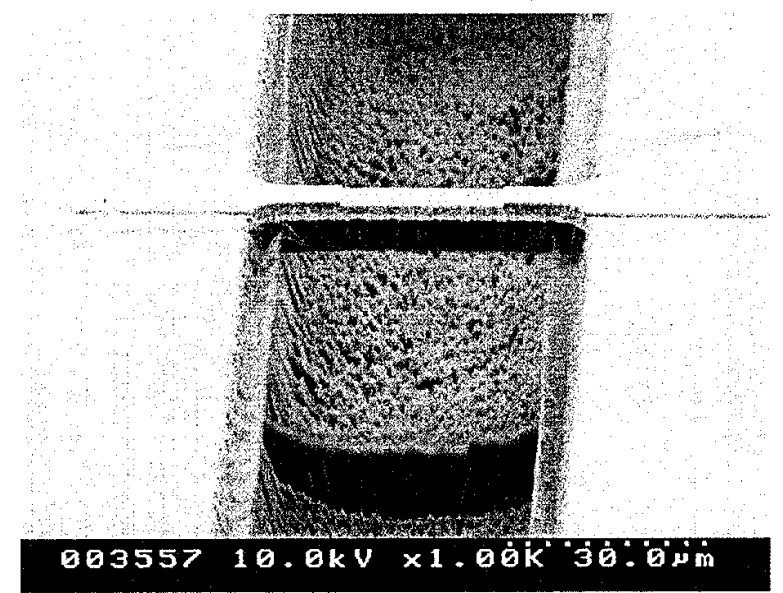

Figure 3. 50- $\mu \mathrm{m}$ beam over 30- $\mu \mathrm{m}$ deep trench.

A detailed description of the PZT material properties and resonator fabrication process used for the reported devices can be found in references [13-15]. The only significant change from previously reported fabrication techniques is that the devices reported here were suspended from the top by an isotropic RIE process, whereas previous techniques used $\mathrm{Si}$ deep reactive-ion etching to release the structures from the backside.

\section{Resonator Operation}

The constitutive equations for the plane stress case with an electric field applied in the $z$-axis can be shown in the following reduced tensor form $[\mathbf{1 6}]$.

$$
\begin{aligned}
& S_{1}=s^{E}{ }_{11} T_{1}+d_{31} E_{3} \\
& D_{3}=d_{31} T_{1}+\varepsilon^{T}{ }_{33} E_{3}
\end{aligned}
$$

In this equation, $S$ is the strain, $s$ is elastic compliance, $T$ is stress, $\mathrm{d}$ is piezoelectric coefficient, $\mathrm{E}$ is electric field, $\mathrm{D}$ is electric displacement, and $\varepsilon$ is the permittivity.

The input drive voltage generates an electric field between the top and bottom electrode and induces a strain in the drive side of the resonator through the direct piczoclcctric cffect. This strain results in a concentrated moment applied at the terminal edge of the input electrode. A bending moment results from the offset of the piezoelectric from the beam neutral axis. When the input voltage frequency is near the fundamental frequency of the beam, resonance occurs in the beam amplifying the piezoelectric strain on the sense side of the resonator. This strain induces a current in the sense circuit at the resonant frequency of the beam through the converse piezoelectric effect.

Figure 4 shows the equivalent circuit [6] for the resonator structure which consists of three components: a drive circuit, a sense circuit, and a feed-through capacitance. The middle ground is the bottom electrode and the input to the drive circuit and the output to the sense circuit are the top electrodes. In the circuit, $C_{p}$ is the static capacitance, $C_{f}$ is the feed through capacitance, and the $L_{x}, R_{x}$, and $C_{x}$ branch is the motional arm of the circuit that produces a series resonance caused by the mechanical vibrations. The mechanical vibration from the drive circuit induces a current in the sense circuit that is reduced by the mechanical coupling factor $\chi_{21}$.

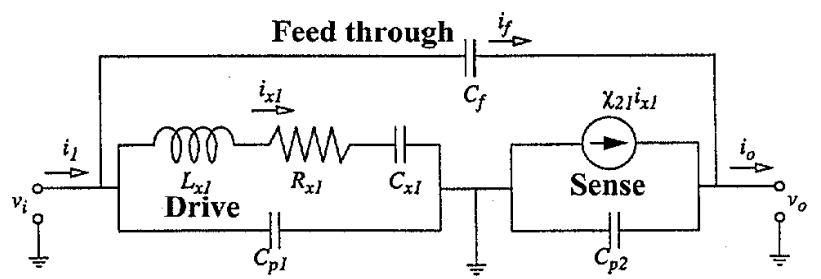

Figure 4. Equivalent circuit.

\section{EXPERIMENTAL SETUP}

The experimental setup included an HP3577 vector network analyzer, an MMR vacuum probe station, and a computer for clectronic data storage. The HP3577 network analyzer provided the drive signal and recorded the scattering parameter $S_{21}$ (ratio of the output to the input, assuming that the output is matched) magnitude and phase for the resonators. The MMR vacuum probe station was used for contacting the top and bottom electrodes. A single ground probe was used since the bottom electrode extends under the entire device. The two remaining probes were placed on the drive and sense top electrodes. The MMR probe station also included a vacuum kit that allowed pressures to be varied from 20 mTorr to ambient. A CLC 109 unity gain operational amplifier was connected at the output port of the MMR probe station due to the impedance mismatch between the $50-\Omega$ test setup and the high impedance of the devices. HP VEE5.0 software was used to dump the data to a text file for electronic storage and analysis.

\section{EXPERIMENTAL RESULTS}

Table 2 shows the dimensions of the reported devices and their designed and actual operating frequencies. There was some variation from the designed theoretical value. We believe that much of the difference was caused by the residual stresses in the clamped-clamped beam structure and the fact that the platinum layers were not considered in the theoretical calculation of the designed frequency and some bulk material properties were used for the calculation. 
Table 2. Theoretical and actual resonant frequencies.

\begin{tabular}{|c|c|c|c|}
\hline $\begin{array}{c}\text { Length } \\
(\mu \mathrm{m})\end{array}$ & $\begin{array}{c}\text { Width } \\
(\mu \mathrm{m})\end{array}$ & $\begin{array}{c}\text { Designed } \\
\text { frequency }\end{array}$ & $\begin{array}{c}\text { Actual } \\
\text { frequency }\end{array}$ \\
\hline 400 & 20 & $59 \mathrm{kHz}$ & $171 \mathrm{kHz}$ \\
\hline 200 & 20 & $236 \mathrm{kHz}$ & $435 \mathrm{kHz}$ \\
\hline 80 & 16 & $1.5 \mathrm{MHz}$ & $1.9 \mathrm{MHz}$ \\
\hline 50 & 10 & $3.8 \mathrm{MHz}$ & $3.6 \mathrm{MHz}$ \\
\hline 25 & 6 & $15 \mathrm{MHz}$ & $9.2 \mathrm{MHz}$ \\
\hline
\end{tabular}

The stress in the overall oxide/Pt/PZT/Pt stack prior to fabrication was measured to be $80 \mathrm{MPa}$ tensile, but at the time of this paper, the actual stress gradient for the various length devices was unknown. An overall tensile stress in the beam should lead to an increase in the resonant frequency as given by the following relation for the fundamental bending mode of a beam subjected to an axial force $\mathrm{N}[17]$.

$$
\omega_{1 n}=\left(\frac{4.73}{L}\right)^{2} \sqrt{\frac{E I}{\rho L}\left(1+0.295 \frac{N L^{2}}{12 E I}\right)}
$$

In this equation, $\omega_{n}$ is the natural resonant frequency, $L$ is beam length, $E$ is effective beam elastic modulus, $I$ is effective beam moment of inertia, $\rho$ is density, $A$ is cross-sectional area, and $N$ is the applied force. This does explain the lower than designed frequency of the 50- and $25-\mu \mathrm{m}$ devices and studies are ongoing.

Figure 5 shows the $S_{21}$ response of an $80-\mu \mathrm{m}$ long resonator operating in a 20 -mTorr vacuum. Its resonant frequency is 1.87 $\mathrm{MHz}$ with a 90 degree phase shift at the resonant peak and a measured loaded $Q$ of 1212 . When the pressure was raised to atmosphcric, the loaded $Q$ reduced to 430 and there was a flattening of the resonant peak due to the increased damping caused by the air flow around the beam. Figure 6 shows the same device operating in air but over a $200-\mathrm{kHz}$ span.

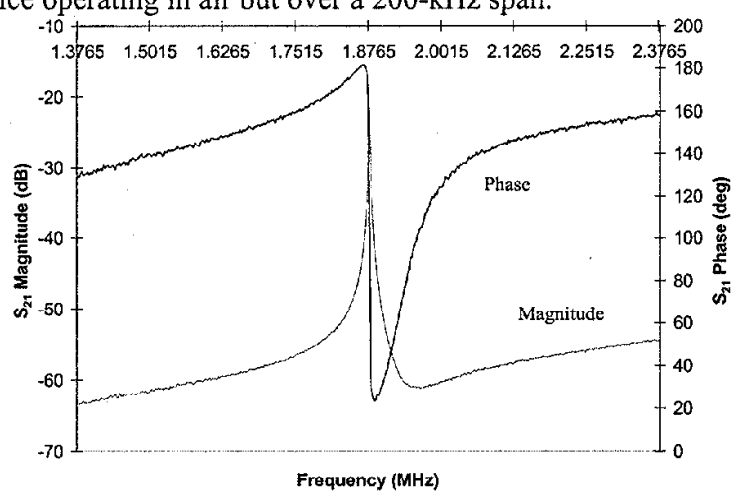

Figure 5. 80- $\mu \mathrm{m}$ resonator at $20 \mathrm{mTorr}$.

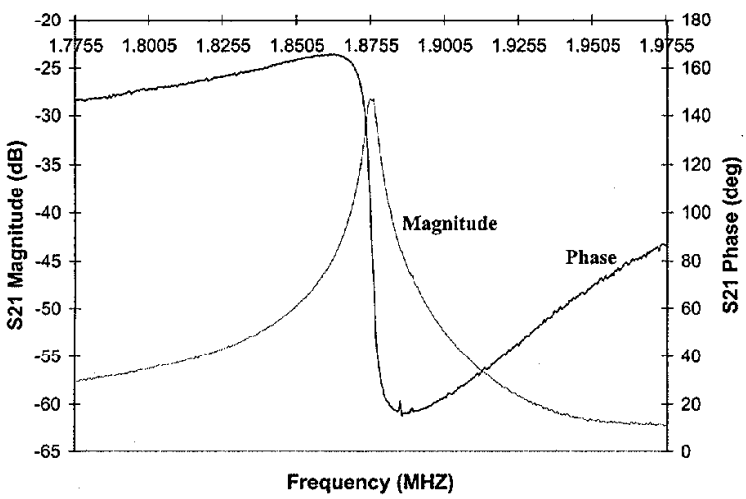

Figure 6. 80- $\mu \mathrm{m}$ resonator at atmosphere.
This same effect was observed for the 400-, 200-, and 25- $\mu \mathrm{m}$ long resonators. Table 2 shows the resonant frequencies of these devices and figure 7 shows the loaded Qs for both the in-air and under 20-mTorr vacuum measurements. The loaded Qs in air appear to be fairly constant between 400 and 500 over the range of devices tested. The loaded $Q$ s for the devices tested under vacuum are much higher ( 3400 at $170 \mathrm{kHz}$ ). The $Q$ for the in-vacuum measurements decreases rapidly at low frequencies but then levels off at approximately 1000 as the frequency is increased above 1 to $3 \mathrm{MHz}$.

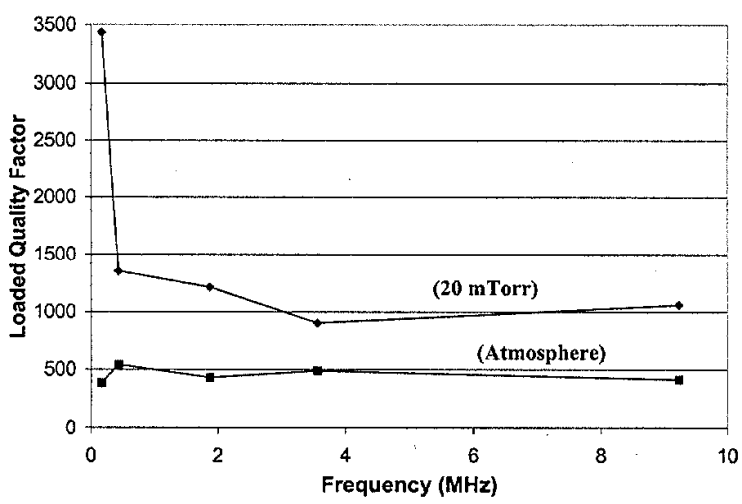

Figure 7. Loaded Qs for resonators measured in air and at 20-mTorr.

Figure 8 shows the effect of the vacuum pressure on the $S_{21}$ measurements for the same $80-\mu \mathrm{m}$ resonator. As the device is brought under vacuum, the $Q$ and magnitude of the resonant peak increase until about 1 Torr is reached and then no further increase is observed through $20 \mathrm{mT}$ Trr. These data show that the resonators work well in air but that a vacuum of less than 1 Torr is required for optimum performance.

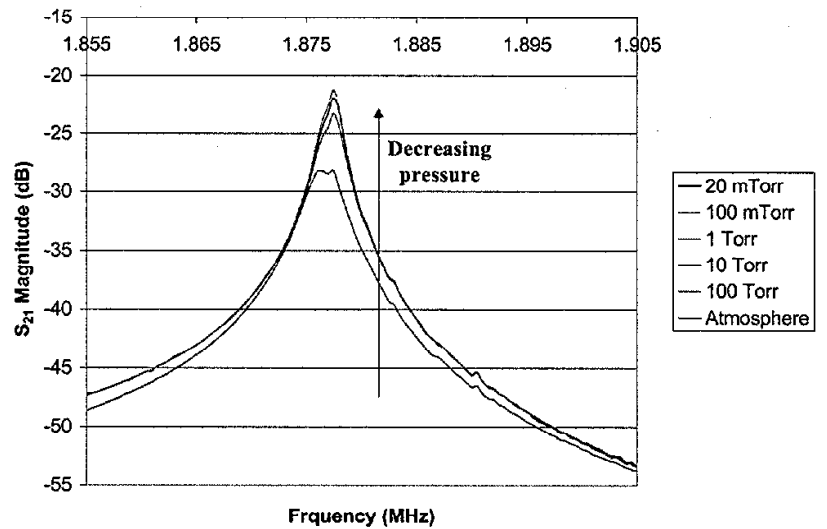

Figure 8. Effect of vacuum pressure on $S_{21}$ Magnitude

The dynamic range was tested by varying the drive voltage from $2.5 \mathrm{mV}$ (minimum voltage the test setup could apply) to 0.8 $V$. Figure 9 shows the results for the $80-\mu \mathrm{m}$ resonator. Evidence of overdriving the resonators was not observed until above $0.25 \mathrm{~V}$ leading to a minimum dynamic range of $40 \mathrm{~dB}$. The resonators do not require a bias voltage and they are not restricted by any gcometrical configuration like the electrode gap for electrostatic devices. 


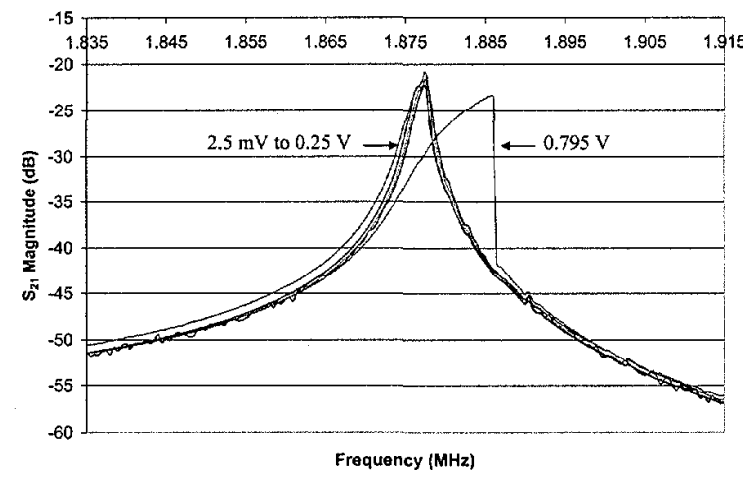

Figure 9. Effect of Drive Voltage on Resonance

Measurements were made without any signal amplification and losses ranged from $-19 \mathrm{~dB}$ at $171 \mathrm{KHz}$ to $-48 \mathrm{~dB}$ at $9.2 \mathrm{MHz}$. The large probe pads ( $100 \times 200$ microns) used in the design resulted in a considerable shunt capacitance to ground. This capacitance induced a large parallel resonance due to finite conductance in the ground electrodes and reduced the signal to noise ratio and obscured the signal at high frequencies. To reduce this effect, large portions of the probe contact pads were removed by running the probe tips across them prior to testing. This resulted in a slight variation in the top electrode area from test to test and a variation in the noise floor for each test. Significant increase and uniformity in the off-frequency rejection is expected to be obtained in the next generation devices, especially at high frequencies, by reducing the contact area.

\section{SUMMARY}

Suspended single-beam microelectromechanical system (MEMS) resonant filters using sol-gel PZT for piezoelectric transduction were demonstrated up to $9.2 \mathrm{MHz}$ with 90 degrees of phase shift at resonance and loaded quality factors greater than 1000 under a $20-\mathrm{m}$ Torr vacuum and greater than 400 in air. Qs up to 3400 were obtained at $171 \mathrm{kHz}$ and the minimum vacuum required for optimum operation was 1 Torr.

The resonators do not require a bias voltage and they are not restricted by any geometrical configuration like the electrode gap for electrostatic devices. The minimum drive voltage used was 2.5 $\mathrm{mV}$ which is the test setup minimum and they exhibited a minimum dynamic range of $40 \mathrm{~dB}$.

\section{ACKNOWLEGEMENTS}

The authors would like to thank Dr. Matthew Ervin for his usual excellent SEM work and Eugene Zakar and Ronald Polcawich for their help in the starting wafer fabrication and bulk film-stack stress measurements.

\section{REFERENCES}

[1] C.T.-C. Nguyen, "Micromechanical resonators for oscillators and filters," Proceedings IEEE Int. Ultrasonics Symp., Seattle, WA (1995) pp. 489-499.

[2] C.T. Nguyen, "Micromechanical Devices for Wireless Communications," Proceedings of the 1998 IEEE Micro Electro Mechanical Systems Workshop, Heidelberg, Germany, (1998). [3] L. Lin, R.T. Howe, A.P. Pisano, "Microelectromechanical Filters for Signal Processing," Journal of Microelectromechanical Systems, 7, (1998).
[4] J.W. Weigold, S. W. Pang, "Fabrication of Thick Si Resonators with a Frontside-Release Etch-diffusion Process," Journal of Microelectromechanical Systems, 7, (1998).

[5] J.J. Yao, N.C. MacDonald, "A Micromachined, Single-Crystal Silicon, Tunable Resonator," J. Micromech. Microeng. 6 (1996).

[6] D.L. DeVoe, "Thin Film Zinc Oxide Microsensors and Microactuators," Ph.D. Dissertation, University of California, Berkeley, Fall (1997).

[7] P. Luginbuhl, Sensors and Actuators, 54 (1996).

[8] C. Lee, Trans. Ultrasonics, Ferroelectrics, and Frequency

Control, 43 (1996).

[9] M.A. Dubois, Trans. Ultrasonics, Ferroelectrics, and

Frequency Control, Sept. (1998).

[10] P. Muralt, Sensors and Actuators, 53 (1996).

[11] E. Zakar, M. Dubey, B. Piekarski, J. Conrad, R. Piekarz, R.

Widuta, "Process and Fabrication of a Thin Film PZT Pressure

Sensor," Presented at the American Vacuum Society $46^{\text {th }}$

International Symposium and to be published in J. Amer: Vac.

Soc., (2000).

[12] E. Zakar, M. Dubey, B. Piekarski, R. Piekarz, J. Conrad, R. Widuta, "Study of PZT Film Stress in Multilayer Structures for MEMS Devices," proceedings of MRS 1999 Fall Symposium, Boston, MA (1999).

[13] S. Trolier-McKinstry, "Manufacturable Sol-Gel PZT Films for Microsensors and Microactuators," Final Technical Report,

U.S. Army Contract DABT63-95-C-0053, (1998).

[14] R.J. Zeto, B.J. Rod, M.H. Ervin, R.C. Piekarz, S. TrolierMcKinstry, T. Su, and J.F. Shepard, "Dry Etching of Sol-Gel

PZT," Proceedings of Material Sci of MEMS Devices, 546, pg 159-164, (1998).

[15] B. Piekarski, M. Dubey, D. DeVoe, E. Zakar, R. Zeto, J. Conrad, R. Piekarz, M. Ervin, "Fabrication Of Suspended Piezoelectric Microresonators," Integrated Ferroelectrics, (1999), 24 , pp 147-154.

[16] T. Ikeda, Fundamentals of Piezoelectricity, Oxford Science Publications, New York NY (1996).

[17] H. Tilmans, M. Elwenspoek, J. Fluitman, "Micro resonant force gauges," Sensors and Actuators A, 30, 35-53, (1992). 